\title{
Un rendez-vous manqué ? la difficile institutionnalisation de l'évaluation des lois fédérales en Belgique
}

\section{Joël Ficet}

Université Libre de Bruxelles, CEVIPOL Campus du Solbosch, CP124, 44 Avenue Jeanne, 1050 Bruxelles

\section{Résumé}

L'évaluation des lois fédérales en Belgique est aujourd'hui en situation de paralysie, en dépit de la création d'un «Comité parlementaire chargé du suivi législatif» bicaméral en 2007. La première raison en est l'ambiguiité de la notion d'évaluation législative, qui oscille entre analyse de l'efficacité et appréciation formelle de la rédaction des lois d'une part, entre évaluation ex ante et ex post de l'autre. L'autre raison en est la situation de tension entre les deux chambres du parlement, qui a culminé en 2014 avec le succès électoral des nationalistes flamands qui souhaitent abolir le Sénat.

(c) 2018 IDMP/Lavoisier SAS. Tous droits réservés

Mots clés : évaluation, analyse d'impact, législation, parlement, Belgique.

\section{Abstract}

A missed appointment? The difficult institutionalization of the evaluation of federal laws in Belgium. Legislative evaluation at a federal level in Belgium is currently to a halt, despite the creation of a bicameral "Parliamentary committee in charge of legislative follow-up" in 2007. The first cause of this paralysis is the ongoing competition between various methodologies of legislative evaluation, which alternate between assessment of the effectiveness or of the formal quality of the laws on the one hand, between ex ante and ex post analysis on the other. The other reason is the increasing tension between the two chambers of the Parliament, especially after 2014 due to the rise of the Flemish nationalists whose objective is to suppress the Senate.

๑ 2018 IDMP/Lavoisier SAS. Tous droits réservés

Keywords: evaluation, impact analysis, legislation, parliament, Belgium.

\footnotetext{
*Auteur correspondant : Joel.Ficet@ulb.ac.be doi :10.3166/pmp.35. 2018.0009 @ 2018 IDMP/Lavoisier SAS - Tous droits réservés.
} 


\section{Introduction}

L'objectif de cet article est de proposer une étude du lent et difficile essor de l'évaluation législative au niveau des autorités fédérales en Belgique. L'évaluation législative peut être définie comme l'analyse prospective ou rétrospective des impacts et/ou de la qualité formelle des normes juridiques délibérées et adoptées par les Parlements. Elle est parente proche de l'évaluation des politiques publiques, dont l'institutionnalisation dans différents contextes nationaux a fait l'objet de nombreuses études comparées (Furubo, Rist et Sandahl, 2002; Jacob et Varone, 2004; Jacob, 2005 ; Furubo, Jacob et Speer, 2015), mais est généralement mise en œuvre par des organes distincts et, par son objet, peut présenter des défis intellectuels spécifiques. Les politiques publiques se présentent sous la forme d'un ensemble de normes, ressources et instruments d'origines parfois hétérogènes mais destinés à résoudre un même problème social affectant un groupe dans un secteur ou espace géographique donné (Mény et Thoenig, 1987); ce qui circonscrit l'objet de l'évaluation de politiques publiques est donc l'enjeu qu'elles visent à traiter. L'évaluation législative, quant à elle, s'attache à un texte (et éventuellement à ses décrets d'application). L'évaluateur peut dès lors se heurter à un problème de focale : les lois se donnent souvent des objectifs multiples (et parfois contradictoires) recouvrant plusieurs programmes; à l'inverse, elles peuvent encadrer des relations sociales sans formuler explicitement d'objectif à atteindre (en matière pénale, notamment); elles s'appliquent généralement à l'échelle d'un territoire et non d'un seul groupe-cible; elles ont le plus souvent vocation à la permanence, à l'opposé d'un programme censé s'interrompre ou s'adapter lorsque le problème social qu'il traite disparaît ou évolue (Haarhuis et Niejmeyer, 2009; Karpen, 2002; Bergel, 1995)... Par ailleurs, les lois ne sont pas purement instrumentales : débattues publiquement et solennellement, elles remplissent des fonctions symboliques implicites (expression d'un consensus, mise en avant d'un problème comme priorité gouvernementale, affirmation de principes supérieurs...) qui ne peuvent être appréhendées sous l'angle de l'efficacité (Bussman, 2010).

Une autre particularité de l'évaluation législative par rapport à celle des programmes publics est l'inclusion fréquente, aux côtés des critères d'efficacité ou d'efficience, de critères relevant de la «qualité » technique de la loi (Flückiger, 2008), ce qui renvoie notamment à la simplicité de la rédaction, à la cohérence interne des textes, à la suppression des actes redondants ou désuets, à la mise en conformité avec les normes supérieures, etc. Luzius Mader établit ainsi un lien entre l'évaluation législative et la « légistique » traditionnellement enseignée dans les facultés de droit (Mader, 2001). Plus précisément, il la rattache à une légistique matérielle, censée apporter des outils méthodologiques pour une meilleure élaboration du contenu des lois; il l'oppose à la légistique formelle, qui s'occuperait de la bonne rédaction et de la structure des lois. Toutefois, comme le montre le cas belge, la distinction n'est pas absolue dans la pratique parlementaire et le choix des critères de l'évaluation législative a des implications proprement politiques, l'attachement à la qualité formelle de la loi au détriment de sa pertinence tendant à neutraliser le potentiel subversif de l'évaluation.

Mais la principale spécificité de l'évaluation législative, d'un point de vue de science politique, est qu'elle intervient dans les relations entre parlements et gouvernements (Duprat, 1998). En tant que source d'information pour le législateur, elle constitue un instrument de renforcement du contrôle démocratique sur l'exécutif (Chevallier, 1995). La prépondérance des gouvernements, en effet, s'appuie sur la monopolisation de l'expertise en matière 
d'action publique, ainsi que sur une connaissance supérieure des mécanismes internes de l'administration. La disponibilité d'une information exhaustive redistribue partiellement les chances d'action entre les deux pouvoirs, et renforce la capacité des parlements à établir publiquement des responsabilités politiques et sanctionner le gouvernement; elle donne une plus grande pertinence et une plus grande crédibilité à leurs initiatives législatives; elle peut également donner un poids supplémentaire aux questions parlementaires (De Peuter, Pattyn et Speer, 2015; Zwaan et al., 2016). L'évaluation législative peut encore affecter l'équilibre entre les deux assemblées d'un même système parlementaire; les cas français et belge montrent à cet égard la fragilité d'organes d'évaluation bicaméraux, aisément paralysés lorsque les chambres sont en conflit. Cette interférence potentielle entre la logique de l'évaluation et les interactions entre autorités constitutionnelles est à prendre en compte pour l'étude du développement de l'évaluation des lois et, en ce qui concerne la Belgique, de son très inégal succès.

Pour opérer cette étude, nous ne prêterons pas uniquement attention aux résultats de l'institutionnalisation de l'évaluation législative, à savoir le statut et les missions des organes en charge de celle-ci. Nous appréhendons en effet ici le concept d'institutionnalisation dans une perspective dynamique, comme un processus politique par lequel l'évaluation législative est mise en forme, légitimée, appropriée et finalement codifiée et routinisée (Lagroye et Lacroix, 1992; Lacouette-Fougère et Lascoumes, 2013). Nous nous appuyons sur des sources parlementaires ainsi que sur des entretiens avec quelques acteurs-clés (qui ont toutefois demandé à ne pas être cités) pour cette analyse d'ordre socio-historique de la genèse des dispositifs d'évaluation des lois, genèse qui commence au tout début des années 1990.

La première expérience notable d'évaluation législative en Belgique est en effet l'instauration d'une commission d'évaluation de la loi du 3 avril 1990 sur l'interruption volontaire de grossesse, une innovation qui sur le moment visait à pacifier les conflits politiques intenses entre partis laïques et catholiques autour de la réforme (Mabille, 1990; Swennen, 2001). Cette commission composée de médecins et spécialistes en la matière fonctionnera toutefois plutôt comme un simple organe de suivi et s'abstiendra de présenter des recommandations de modification du régime de l'IVG. Mais parallèlement à cette initiative ponctuelle, la décennie 1990 apparaît comme une phase d'effervescence où des projets visant à créer un dispositif permanent d'évaluation sont élaborés tant par les gouvernements successifs que par des élus des deux chambres. La loi-programme du 10 février 1998 pour la promotion de l'entreprise indépendante institue ainsi une première forme d'analyse d'impact, sous l'apparence de « fiches administratives d'impact », élaborées sous la supervision d'une Agence pour la simplification administrative (ASA), et destinées à prévenir les éventuelles charges imposées aux entreprises par tout projet de loi ou de règlement. Par la suite, cet instrument sera généralisé et étendu à d'autres thématiques, telles que le développement durable et l'égalité de genre. Il ne s'agit néanmoins là que d'une évaluation ex ante, qui par surcroît ne s'applique qu'aux avant-projets de loi rédigés par le gouvernement. Après de nombreux débats et tractations, la loi du 25 avril 2007, finalement, instaure un « Comité parlementaire chargé du suivi législatif », organe bicaméral chargé d'évaluer les lois fédérales en vigueur depuis au moins trois ans selon un double critère de qualité juridique et d'adéquation des lois aux situations qu'elles régissent. Pour accomplir sa mission, le Comité s'appuie sur les rapports des institutions judiciaires et du Conseil d'État, mais aussi sur les requêtes que peuvent déposer sur son site internet les citoyens, les administrations et les entreprises. Le bilan de l'action du Comité est 
toutefois limité, et ses travaux sont interrompus depuis les élections législatives de mai 2014. L'évaluation des lois par le Parlement, par contraste avec l'analyse d'impact des avant-projets de loi par le gouvernement, apparaît de la sorte comme un échec.

En vue d'une explication de la diversité et de la complexité des trajectoires de l'institutionnalisation de l'évaluation législative, nous posons quatre hypothèses. La première est que le développement de l'évaluation législative s'inscrit en Belgique dans un mouvement idéologique d'inspiration libérale, hostile à la régulation et à l'interventionnisme étatique ; ses plus fervents promoteurs ont d'ailleurs souvent été des représentants des partis libéraux néerlandophone et francophone. Cette inspiration constitue, dans une large mesure, la raison pour laquelle l'évaluation législative a pris son premier envol dans le cadre de la politique de simplification administrative, explicitement destinée à protéger le marché et les citoyens contre la pesanteur de la législation. La seconde hypothèse met l'accent sur l'influence du discours des organisations internationales, au premier rang desquelles l'OCDE et l'Union Européenne. L'évaluation ex ante par l'exécutif (ou analyse d'impact), plus particulièrement, a progressé en résonance avec les engagements internationaux du pays, par exemple en matière d'égalité entre hommes et femmes. Cette influence est par contre peu présente dans les délibérations du Parlement en vue de mettre en place, en son sein, un dispositif permanent d'évaluation des lois. La lecture des multiples propositions en ce sens, étalées sur plus de quinze ans, révèle - et c'est là notre troisième hypothèse - que l'un des principaux obstacles à l'adoption d'un tel dispositif est la tension entre les deux dimensions de l'évaluation législative évoquées plus haut, celle de la qualité formelle des textes et celle de leurs effets ; les parlementaires semblent ainsi avoir constamment hésité entre la première, jugée trop peu ambitieuse, et la seconde, par trop « politique ». Enfin, il est inévitable de relever la détermination de l'institutionnalisation de l'évaluation par les traits systémiques des régimes politiques, tels que le rôle des partis ou les relations entre Parlements et gouvernements (Jacob, 2005). Nous soulignerons dans le cas particulier de la Belgique, le poids de deux facteurs, qui tendent à se recouvrir dans les dernières années : la concurrence entre les deux chambres du Parlement, et les omniprésentes tensions intercommunautaires entre partis francophones et néerlandophones.

\section{Aux origines de l'évaluation des lois en Belgique, une vision politique libérale}

Le constat d'une telle inspiration libérale, peut-on noter en premier lieu, n'est pas singulier à la Belgique. Dans la plupart des pays voisins, le développement de l'évaluation des lois a accompagné la mise en place de réformes néo-managériales et de politiques favorables au marché. Cette évolution générale de la pensée sur les missions de l'État se traduit dès lors par une méfiance générale vis-à-vis de la production de nouvelles normes juridiques, toujours soupçonnée d'imposer des contraintes administratives abusives aux entreprises et aux citoyens. Il n'est à cet égard pas étonnant que la première forme d'évaluation ex ante des projets de loi et de règlement ait été, comme on l'a noté plus haut, instaurée par une loi-programme sur la promotion des entreprises.

Cette orientation est confirmée par le gouvernement dirigé par le libéral Guy Verhofstadt (juillet 1999-mars 2008), qui décide au début des années 2000 de systématiser la procédure de la « fiche d'impact ». Le gouvernement décide au début des années 2000 de la systématiser. 
Ceci conduit à la naissance en 2004 du « Test Kafka » (un nom au combien révélateur!), sous l'égide du secrétaire d'État à la simplification administrative Vincent Van Quickenborne (membre comme Guy Verhofstadt du parti libéral flamand VLD). Cette nouvelle procédure impose que chaque texte soumis au conseil des ministres (y compris les avant-projets de loi) comprenne une annexe répondant aux questions suivantes : le projet a-t-il un impact sur les charges administratives qui pèsent sur les citoyens, les entreprises et/ou le secteur marchand? Le projet vise-t-il à supprimer ou à réduire ces charges administratives ? Quelles charges administratives nouvelles ou complémentaires le projet implique-t-il? Quelles mesures envisage-t-on pour éviter, le plus possible, des charges administratives nouvelles ou complémentaires? Ces réponses sont censées être par la suite communiquées aux parlementaires afin de nourrir leurs délibérations.

La recherche de simplification administrative est, dans les projets de l'exécutif, connectée à l'enjeu de l'évaluation des lois. Verhofstadt associe ainsi les deux thématiques dans sa déclaration de gouvernement, prononcée le 14 juillet 1999 devant le parlement : « La surabondance de lois et règlements ébranle la sécurité juridique et le sentiment de justice des citoyens. Trop de dispositions légales et réglementaires freinent la créativité des citoyens et des entreprises et leur sens de l'initiative. Le gouvernement fédéral diminuera de $10 \%$, au cours des deux années à venir, la charge administrative des citoyens et des entreprises pour réduire d'un quart l'ensemble des formalités et obligations d'ici la fin de la législature (...) Le gouvernement, avec le Parlement et en collaboration avec le bureau de coordination du Conseil d'État, procédera à une évaluation de la législation. Celle-ci est un moyen d'améliorer l'efficience, l'adhésion citoyenne et la qualité juridique de la législation. Cette évaluation de la législation permettra de juger leur fonctionnement et leur efficacité (sic), de sorte qu'elles puissent être revues et, au besoin, abrogées $»^{1}$. Les deux démarches reposent ainsi, dans cette perspective, sur le postulat commun d'une inefficacité et d'un excès de droit.

Mais l'intention d'institutionnaliser l'évaluation législative ex post en lien avec le Parlement, toutefois, ne se concrétise pas avant 2007 et la mise en place du Comité de suivi législatif. À nouveau, l'inspiration politique du projet est clairement libérale. L'auteur de la proposition de loi instituant le Comité, le député Daniel Bacquelaine (libéral francophone), insiste ainsi sur la nécessité de prévenir contre une «inflation légistique » qui « décourage les citoyens et les structures telles que les petites et moyennes entreprises ou les administrations »; dans ce but, il conviendrait de "simplifier l'enchevêtrement des textes et des législations, supprimer les doublons, abroger de vieux textes désuets ». Mais il présente également le Comité comme une «avancée de la démocratie directe »: «La loi offre effectivement un nouveau droit au citoyen. Désormais, il pourra s'adresser directement à nous par voie de requêtes (...) On écoutera aussi les petites entreprises, les petites administrations qui sont des structures parmi les plus exposées aux difficultés liées à l'inflation et à la complexité croissante de la production normative $»^{2}$. La possibilité de saisine du Comité par les individus ou les entreprises est ainsi présentée, devant la presse, comme une arme au service de la société civile contre les dérives du Parlement ${ }^{3}$.

\footnotetext{
${ }^{1}$ Doc. Chambre, 20/1, 14 juillet 1999, p. 13.

${ }^{2}$ Chambre, Séance plénière, 22 mai 2004, pp. 36-37.

3 « Les citoyens pourront bientôt signaler les lois qu'ils estiment inutiles », La Libre Belgique, 8 avril 2013.
} 
Cette orientation idéologique, déterminante aux origines de l'évaluation des lois, a encore des effets sur la pratique actuelle : l'analyse d'impact des lois et règlements (AIR), par exemple, est toujours coordonnée par l'Agence de simplification administrative. Il n'est par ailleurs pas innocent que le développement de l'AIR prenne sa source dans les travaux de l'OCDE, qui au cours des années 1990 lance une offensive conceptuelle sur le thème de la Better Regulation en mettant particulièrement l'accent sur la réduction des contraintes sur les entreprises (OCDE, 1997). Au long des années suivantes, les recommandations de diverses organisations internationales ont conditionné de façon similaire le développement de l'évaluation des lois ex ante.

\section{L'institutionnalisation de l'évaluation ex ante des projets de loi : l'impact des recommandations internationales}

Fondée sur une vision normative du cycle des décisions publiques, généralisant une appréciation économiciste des coûts et bénéfices des réglementations et valorisant le savoir objectif des experts sur l'autorité des élus, l'évaluation ex ante ou analyse d'impact est souvent interprétée comme un des avatars de la croyance en une approche scientifique et objective du politique (Radaelli et De Francesco, 2010). Toutefois, face aux échecs et apories de l'evidence-based policy-making, d'autres explications du succès de l'AIR ont été apportées. Radaelli en mentionne ainsi trois facteurs : l'influence du nouveau management public, la quête d'un meilleur contrôle des agences administratives par les gouvernements, et l'importation de modèles internationaux et étrangers à des fins de légitimation des autorités nationales (Radaelli, 2009). L'analyse d'impact à la belge, toutefois, est plus qualitative qu'économique; bien que son apparition coïncide avec de vastes réformes néomanagériales au niveau fédéral (Bouckaert et Pollitt, 2011), elle s'est développée selon un calendrier séparé; enfin, elle apparaît plus destinée à informer les cabinets ministériels (qui traditionnellement monopolisent la rédaction des textes) ou les parlementaires qu'à encadrer l'activité d'agences autonomes à l'américaine. Les circonstances de la naissance du test Kafka suggèrent que l'émergence de l'AIR en Belgique relèverait plutôt de la troisième explication évoquée par Radaelli, qu'il caractérise comme relevant d'une forme d' « action symbolique » (Radaelli, 2009) : une appropriation rituelle de concepts et instruments transnationaux, destinée à démontrer l'apolitisme et la rationalité des orientations gouvernementales, mais au total largement déconnectée de la réalité de la prise de décision. Et en effet, dès 2004, à l'occasion des débats parlementaires précédant l'institution du Comité de suivi législatif, le secrétaire d'État Van Quickenborne admettait que le test Kafka mis en place deux ans plus tôt était devenu une «clausule stylistique». Selon ses propres mots, «le test est souvent mentionné pro forma, mais aucun examen préalable n'a été mené »". Malgré ce constat pessimiste, l'institution du test Kafka n'est que le début d'une expansion graduelle du champ de l'analyse d'impact des lois, au gré des contraintes ou opportunités fournies par les organisations et conventions internationales. Par un processus de policy transfer (Dolowitz et Marsh, 2000), en effet, les gouvernements successifs ont ainsi intégré à l'évaluation ex ante des lois et règlements de nouveaux critères, reproduisant à plusieurs

${ }^{4}$ Doc. Chambre, 51 0029/009, 15 mars 2004, Annexe 1, p. 40. 
reprises la technique du test thématique jusqu'à l'adoption d'un instrument intégré par la loi du 15 décembre 2013.

La première étape de ce processus de bricolage institutionnel est l'introduction, en janvier 2007, d'une « évaluation d'incidence des décisions sur le développement durable » (EIDDD). Ceci correspond au mouvement d'insertion de la notion de développement durable dans la gouvernance du pays, dont le point le plus marquant est la reconnaissance du concept comme objectif de la politique générale de la Belgique par la réforme constitutionnelle du 25 avril 2007. Comme son nom l'indique, l'EIDD vise à anticiper les conséquences globales et de long terme des législations, en termes notamment de changements environnementaux (Kegels et Debeuckelaere, 2000). Elle repose sur un questionnaire reprenant 33 items (impact sur la consommation d'énergie, la qualité de l'air, la mobilité, la biodiversité...). Initialement dénuée de force juridique, elle est peu après dotée d'un ancrage légal par la loi du 30 juillet 2010 .

La seconde étape est l'annonce d'un «Test Gender» par la loi du 12 juin 2007 visant au contrôle de l'application des résolutions de la conférence mondiale sur les femmes réunies à Pékin en septembre 1995 et intégrant la dimension du genre dans l'ensemble des politiques fédérales. L'intitulé complet de la loi est parlant : il s'agit, sous l'influence du discours et des normes des organisations internationales (l'Union européenne et le Conseil de l'Europe développent simultanément leurs propres programmes de promotion du « Gender mainstreaming »), d'introduire la perspective de l'égalité hommes/femmes de manière systématique dans la prise de décision fédérale. Le Test Gender, dans cette perspective, tend à une évaluation préalable de l'impact de tout projet d'acte législatif ou réglementaire ou budgétaire sur la position socio-économique relative des hommes et des femmes.

Ces deux procédures partagent de nombreux points communs. Issues de concepts élaborés au niveau transnational, elles correspondent à des obligations transversales en deux sens s'appliquant à tous les secteurs d'intervention de l'État et à chaque phase du cycle de l'action publique. Elles conduisent dès lors logiquement, dans le cadre d'un modèle de planification, à une évaluation ex ante de tous les textes législatifs et réglementaires. Ces innovations ont également été facilitées par les objectifs qui les sous-tendent. La protection de l'environnement et l'égalité de genre sont des thématiques consensuelles dans le pays, et chaque force politique peut retirer des bénéfices de leur promotion. Aussi, les seules contestations de ces mesures n'ont pas porté sur leur principe, mais sur les conditions de leur efficacité; lors de la discussion à la Chambre de la proposition de loi visant à la codification de l'EIDD, la seule critique viendra du groupe écologiste, pour lequel la rédaction proposée par deux députés démocrates-chrétiens ne donne pas aux résultats de l'évaluation une force contraignante suffisante ${ }^{5}$.

Mais la multiplication des « tests » ne s'arrête pas là, et deux autres dispositifs similaires sont adoptés dans les années qui suivent. Le premier est inspiré par le Small Business Act européen de 2008 (SBA), en application duquel le gouvernement crée un « test PME », sous forme - à nouveau - d'un questionnaire obligeant les administrations fédérales à mesurer l'impact probable de toute nouvelle législation et réglementation sur les PME, et les incitant à réfléchir sur les moyens de soutenir celles-ci à tous les niveaux. Ce test transpose une procédure semblable suivie par la Commission Européenne.

\footnotetext{
${ }^{5}$ Doc. Chambre, 52 2238/004, 8 février 2010, p. 44.
} 
Enfin, la loi du 19 mars 2013 relative à la Coopération belge au développement introduit une analyse d'impact des politiques intérieures et extérieures belges sur les pays en développement, axée sur la question de la cohérence des politiques de développement (CPD) menées par les différentes institutions publiques fédérales ou fédérées. La mise en avant de ce critère de la CPD renvoyait aux évolutions du discours international sur les politiques de développement ainsi qu'à l'introduction du concept dans l'article 208 du Traité de Rome en 2009.

L'institutionnalisation de l'évaluation ex ante en Belgique a ainsi progressé par sédimentation de dispositifs partiels. L'établissement d'un instrument unique, toutefois, semblait inéluctable : d'une part, l'addition de questionnaires ne pouvait qu'alourdir le travail des fonctionnaires et des élus (alors même que l'un des buts initiaux de l'analyse d'impact était la simplification administrative!); d'autre part, les objectifs des différents tests entrent en résonance du fait même de leur transversalité (la simplification administrative participe du soutien aux PME, l'aide au développement est aussi liée à l'égalité de genre...); enfin, l'intégration des instruments belges d'analyse d'impact est recommandée en 2010 par un rapport de l'OCDE (OCDE, 2010). Aussi, la loi du 15 décembre 2013 fusionne finalement ces cinq outils en une seule procédure, le test AIR, qui comporte désormais 21 rubriques et est présenté comme relevant d'un objectif global de développement durable. L'Agence de simplification administrative, au sein de laquelle est créé exprès un Comité d'analyse d'impact, garde sa mission de supervision de la réalisation des AIR. Ceci, de même que la reprise du terme de «test », reflète la nature incrémentale de l'institutionnalisation de l'évaluation ex ante des projets de loi (qui, en l'occurrence, emprunte la même voie que l'évaluation réglementaire) en Belgique.

Il faut toutefois souligner les limites du dispositif. Certaines matières en sont exclues, comme les actes relatifs aux traités internationaux ou aux accords de coopération entre fédération et entités fédérées (régions et communautés), mais également les actes relatifs à « l'autorégulation de l'autorité fédérale » - ce qui peut inclure le statut des fonctionnaires. Or, on conçoit que ce dernier type de normes pourrait utilement donner lieu, par exemple, à une évaluation en termes d'égalité hommes / femmes. De plus, seuls les actes passant en Conseil des ministres sont automatiquement évalués. Ceci laisse de côté les propositions de loi, pour l'élaboration et l'examen desquelles les membres de la Chambre des représentants et du Sénat sont donc privés d'une source précieuse d'information. De fait, simultanément à l'investissement des gouvernements dans des outils d'étude d'impact qui concernent avant tout la réglementation, une réflexion se développe, sur des bases intellectuelles différentes, en vue de la mise en place d'une évaluation des lois par le Parlement.

\section{L'institutionnalisation de l'évaluation parlementaire des lois, de la légistique à l'évaluation ex post}

Les propositions de loi visant à institutionnaliser l'évaluation des lois au sein du Parlement fleurissent à partir du début des années 1990. Ainsi, une proposition de loi instituant une évaluation triennale de la législation est déposée à la Chambre en mai 1992 par le socialiste flamand Johan Vande Lanotte. En mai 1997, une proposition visant à créer au sein des services du Sénat une cellule d'évaluation de la législation est déposée par le démocratechrétien flamand Hugo Vandenberghe. Deux propositions encore sont déposées en 1997 par le nationaliste flamand Geert Bourgeois, l'une visant à créer auprès de la chambre un 
« office des légistes » dont une des missions serait l'évaluation des lois, l'autre relative à la collaboration de la Cour de cassation à l'évaluation de la législation... À ces initiatives sont à ajouter de multiples textes portant sur la simplification, la coordination ou la révision des lois, dont l'objet recoupe partiellement la problématique de l'évaluation législative. Cette effervescence n'a pourtant abouti que difficilement à une véritable innovation politique, le Comité de suivi législatif déjà mentionné plus haut. L'une des raisons de ce délai est un conflit sur le sens même de l'évaluation législative. Une lutte de définition se réouvre en effet à chaque proposition de loi ; elle s'articule autour de deux modèles. Le premier est axé sur la qualité juridique du texte, et s'appuie sur l'expertise des instances juridictionnelles; il restreint dès lors la portée de l'évaluation à des considérations de clarté, de désuétude, de redondance ou de non-conformité avec d'autres normes juridiques. Le second, plus ambitieux, sollicite des savoirs scientifiques en vue d'une véritable évaluation d'impact ainsi que de l'expression d'un jugement politique sur la pertinence du texte concerné. Le Comité de suivi législatif institué en 2007 concilie ces deux visions.

Le premier modèle est parfaitement illustré par la proposition Vande Lanotte de 1992. Les motifs de la proposition mentionnent certes (brièvement) la nécessité «d'adapter la législation à l'évolution de la société », mais c'est surtout l'abrogation des normes désuètes qui est visée ${ }^{6}$. Significativement, le maître d'œuvre pressenti pour ce travail est le Conseil d'État, jugé « le mieux outillé » pour établir, par un rapport annuel, un « relevé des normes juridiques qui sont dépassées en tout ou en partie ou dont l'exécution, l'interprétation ou l'application pose des problèmes ». Par ailleurs, est-il mentionné dans les motifs, «l'objectif n'est pas d'aborder des sujets de haute teneur politique », car "l'évaluation de la politique est une prérogative du Parlement et du gouvernement et ce serait une erreur d'entraîner des instances indépendantes dans ce débat ». Le texte proposé stipule en conséquence en son article 2 que «les normes dont l'adaptation pose un problème politique manifeste sont omises $\gg^{7}$ du champ de l'évaluation triennale. Il apparaît ici clairement que cette nouvelle procédure, dont la mise en œuvre est confiée à un corps de juristes, se limite à des considérations formelles et préserve entièrement la mainmise des forces politiques sur la prise de décision : elle reste donc bien en-deçà d'une évaluation au sens plein du terme, qui implique une analyse critique des objectifs et moyens d'un programme, et la formulation de recommandations. Elle partage l'inspiration d'autres propositions de la même période qui, sans forcément employer le terme d'évaluation, tendent à établir un dispositif de mise en cohérence formelle et/ou de simplification des lois. Parmi celles-ci, la proposition du sénateur Étienne Cerexhe visant à créer une commission nationale chargée de la coordination et de la simplification de la législation $\left(\right.$ mars 1992) ${ }^{8}$ et la proposition du député Hendrik Daems d'instaurer une Commission parlementaire spécifique au sein de la Chambre des représentants (mai 1992), en charge de « supprimer les lois désuètes », d' "actualiser les lois totalement dépassées par l'évolution sociale », d' "assainir radicalement la législation » et d' " arrêter l'inflation de proposition de loi $»^{9} . .$. Les premières réflexions pour une évaluation législative au Parlement la dépolitisent donc, en la cantonnant ostensiblement à un

\footnotetext{
${ }^{6}$ Doc. Chambre, 439 / 1, 7 mai 1992, p. 2. Le rapport de la Commission constituée pour examiner la proposition de loi parle ainsi de « nettoyage » des textes.

기.

${ }^{8}$ Doc. Sénat, 229/1, 9 mars 1992.

9 Doc. Chambre, 439/2, p. 8.
} 
simple constat des défectuosités techniques des textes et en lui associant des organes euxmêmes situés hors du jeu politique, le Conseil d'État et la Cour de Cassation. À l'inverse, elles écartent prudemment toute appréciation de l'opportunité politique des législations ou l'analyse de thématiques controversées.

Le débat fait toutefois un pas en avant avec la proposition de loi Vandenberghe (mai 1997), portant sur la création d'une cellule d'évaluation de la législation au Sénat. L'ambition du texte est au premier regard limitée, mais les motifs de la proposition, particulièrement élaborés, témoignent d'une maturation de la réflexion parlementaire. L'auteur de la proposition tente en effet de définir précisément l'objet de l'évaluation législative en énonçant « dix commandements » pour une bonne législation, qu'il répartit en critères juridiques (sécurité juridique, égalité...), critères « socio-scientifiques » (effectivité, efficacité, effet social...), et critères «mixtes » relevant des deux catégories précédentes (subsidiarité, précision de l'objectif, nécessité... $)^{10}$. Ce faisant, il assume que la future cellule d'évaluation établira un véritable examen critique de la pertinence, des instruments et des impacts de chaque loi, au moyen de méthodes scientifiques. Cette conceptualisation des fondements de l'évaluation législative donne lieu à une large discussion devant la Commission des affaires institutionnelles du Sénat, en présence de magistrats et d'universitaires, au cours de laquelle s'exprime de manière très transparente le clivage souligné plus haut entre une évaluation « technico-juridique » et une évaluation plus « politique ». L'auteur de la proposition estime que, l'évaluation des lois étant une opération impliquant des options politiques, elle incombe naturellement à une des chambres du Parlement. De ceci découlerait l'impératif de créer une cellule spéciale au sein du Sénat, le Conseil d'État n'étant pas à même d'apprécier des éléments tels que l'applicabilité ou l'effet social d'une loi. Un autre orateur, inversement, estime qu'une évaluation par des fonctionnaires du Sénat serait suspecte de partialité. D'autres encore s'élèvent contre l'application de ces critères « socio-scientifiques », qui mettraient en compétition la cellule d'évaluation avec les commissions parlementaires... Les délibérations ne permettent pas de trancher le dilemme : la proposition aboutira effectivement à la mise en place d'un « service d'évaluation » au Sénat, mais celui-ci, notamment par manque de ressources, ne se livre aujourd'hui qu'à des analyses ex ante de la qualité juridique et linguistique des propositions de loi.

Ce travail de mise en forme intellectuelle contribue malgré tout à la matérialisation juridique de l'évaluation législative. En juin 2003, une proposition de loi instaurant une évaluation parlementaire par un comité ad hoc est déposée à la Chambre des représentants par le député Daniel Bacquelaine. Celle-ci reçoit le support de l'exécutif et, réintroduite en tant que projet de loi, conduit après un jeu de va-et-vient entre la Chambre et le Sénat, à la mise en place du Comité de suivi législatif par la loi du 25 avril 2007.

Le Comité est un organe bicaméral composé de onze députés et de onze sénateurs. Il est chargé d'assurer une évaluation des lois fédérales en vigueur depuis au moins trois ans, sous deux angles. Le premier est celui de l'identification des difficultés d'application liées à la complexité, aux lacunes, aux incohérences, aux erreurs et à la désuétude des lois, ainsi que la conformité de celles-ci à la jurisprudence constitutionnelle; on retrouve là des formulations déjà présentes dans des textes antérieurs. Le second critère énoncé est celui de «l'inadéquation » des législations aux situations qu'elles régissent, censée être établie

\footnotetext{
${ }^{10}$ Doc. Sénat, 643/1, 20 mai 1997.
} 
par l'examen de ces derniers du point de vue de l'efficacité, de la proportionnalité, de la transparence et de la cohérence. Le concept n'est pas mentionné dans les articles définissant les missions du Comité, mais les débats parlementaires indiquent clairement que ce test d'inadéquation correspond bien à une évaluation ex post de la pertinence des lois ${ }^{11}$. Une seule limitation à ces ambitieux objectifs : le Comité n'a pas la faculté d'introduire lui-même les propositions de loi qu'il rédige. Il doit pour cela saisir les Commissions parlementaires ou le ministre compétents.

Les travaux parlementaires témoignent donc d'une évolution au moins partielle des perceptions : le Comité de suivi législatif est en effet un compromis entre les deux « modèles » décrits plus haut. Toutefois, l'évolution du vocabulaire ne se traduit pas en résultats concrets. Du fait d'une lenteur à désigner ses membres, ce comité ne se réunit la première fois qu'à l'automne 2011. L'essentiel de ses travaux porte par la suite sur la mise en conformité des lois avec la jurisprudence de la Cour constitutionnelle, notamment sur la base des recommandations de la Cour de Cassation et du Conseil d'État; cette tâche aride est principalement effectuée par les fonctionnaires des assemblées, les séances du Comité n'attirant l'attention que d'un noyau de parlementaires particulièrement motivés. Par ailleurs, la procédure électronique pour le dépôt des requêtes individuelles, qui devaient servir de base à une évaluation plus politique, n'est effective que le 29 avril 2013. Entre cette date et le 31 mars 2014 (fin de la législature), 80 requêtes sont reçues par le Comité, émanant toutes de particuliers (les entreprises ou les administrations n'ont pas exploité ce nouveau droit). Un tiers de ces requêtes ont été jugées irrecevables, qu'elles soient insuffisamment motivées ou qu'elles ne concernent pas la législation fédérale. Les autres ont été classées sans suite (le Comité estimant qu'une modification de la loi n'était pas utile) ou transmises à une autre instance (commission parlementaire compétente, commission des pétitions de la Chambre, ministre concerné, autorités régionales, médiateurs des administrations).

Enfin, le bilan du Comité en termes de production normative est lui-même restreint. Il a au total élaboré et obtenu l'adoption de trois propositions de lois qui se présentent chacune comme un catalogue de ce qu'un des rapports d'activité du Comité décrit lui-même comme des «corrections purement techniques $»^{12}$. Par surcrôit, le fonctionnement du Comité, qui ne s'est pas réuni depuis les élections législatives de mai 2014, est aujourd'hui interrompu. Les raisons de cette paralysie relèvent de notre dernière hypothèse : l'influence de la concurrence entre organes constitutionnels ainsi que des tensions entre communautés francophones et néerlandophones.

\section{L'évaluation législative, otage des querelles communautaires}

L'évaluation des lois ou l'analyse d'impact, de par leurs procédés et leur langage, se veulent des méthodes d'aide à la décision rationnelles et non-idéologiques. L'observation montre toutefois que, dans tous les pays qui les pratiquent, leurs formes et leurs résultats

\footnotetext{
11 Une ambiguïté est toutefois introduite par l'article 7 de la loi, selon lequel le Comité « s'attache à vérifier si les moyens mis en œuvre [par les lois] permettent de produire les effets attendus et d'atteindre les objectifs assignés ». Cette formulation semble plutôt s'appliquer à une évaluation ex ante.

12 Doc. Chambre, 53-1969/3, 24 avril 2014, p. 45.
} 
dépendent des stratégies institutionnelles des acteurs politiques (Radaelli, 2010). L'évaluation législative, notamment, est de manière récurrente perçue comme une arme contre l'exécutif pour des Parlements de plus en plus marginalisés du fait de la complexité croissante et de la transnationalisation de la production juridique.

Cet enjeu transparaît en filigrane (et parfois de manière explicite) dans la discussion des diverses propositions susmentionnées. Toutes partent des mêmes diagnostics : impuissance du législateur face à la multiplication des projets de loi, soumission aux impératifs gouvernementaux, qualité médiocre d'un droit élaboré sur base de compromis politiques, nécessité d'un soutien (en termes d'information, de compétences ou de personnel) pour exercer correctement une fonction de contrôle et pour adapter les lois aux évolutions sociales ... L'institutionnalisation de l'évaluation serait dans cette perspective un des moyens de replacer le Parlement au centre du processus législatif.

Plusieurs voies sont envisagées pour pallier ce manque. Certaines propositions tendent à externaliser la production d'expertise en mettant à contribution des institutions existantes (Conseil d'État, Cour de Cassation) ou à en créer de nouvelles, telle la « commission nationale chargée de la coordination et de la simplification de la législation » (composée d'universitaires et de magistrats) qu'imagine le sénateur Étienne Cerexhe en 1992. D'autres envisagent la mise en place de services ad hoc au sein de l'administration du Parlement, admettant de manière franche l'incapacité des parlementaires à rédiger de bonnes lois. Ainsi, le député Jean-Jacques Viseur (démocrate-chrétien francophone) suggère la création d'un « office des légistes » à la Chambre en octobre 1997; les membres de cet office n'interviendraient toutefois qu'au niveau de l'écriture des lois ${ }^{13}$. Le député Geert Bourgeois, en juin de la même année, propose quant à lui un «collège des légistes », dont l'objet serait de devenir «la clé de voûte de la revalorisation du travail législatif du Parlement $»^{14}$. Composé d'experts juristes, ce collège devait rédiger les propositions de loi à la demande de la Chambre, permettant aux élus de se focaliser sur la direction politique et la définition des objectifs; il était également censé évaluer la législation existante et formuler des recommandations (bien que les modalités ne soient guère détaillées par le texte). La proposition associe clairement la perte d'influence du Parlement au manque d'expertise des élus, et se veut un rappel à l'ordre : «Le Parlement ne pourra recouvrer sa dignité qu'en exerçant pleinement ses prérogatives et en agissant en conséquence. Cela vaut pour son droit de contrôle tout autant que pour le travail législatif. Un parlement conscient de sa mission ne se laisse pas imposer des lois de pouvoirs spéciaux, refuse d'adopter des mauvaises lois présentées comme étant 'à prendre ou à laisser' et rejette, en général, tout travail législatif peu sérieux ou de qualité médiocre $\gg^{15}$. Enfin, une troisième voie est la création d'une commission d'élus dotée de pouvoirs d'évaluation. Concrétisée sous la forme du Comité de suivi législatif, cette approche ne résout toutefois pas la question de l'expertise : les parlementaires sont généralement dénués d'expérience en matière d'évaluation et, bien souvent, ont d'autres priorités.

Mais le nœud des controverses n'est pas, dans le cas belge, la concurrence entre législateur et exécutif. En effet, le système politique belge est un régime de coalition où les rapports de force pertinents en termes de prise de décision s'établissent entre les partis

\footnotetext{
13 Doc. Chambre, 1098/1, 10 octobre 1997.

${ }^{14}$ Doc. Chambre, 1071/1, 10 juin 1997, p. 5.

15 lbid., p. 2.
} 
représentant les « piliers » philosophiques (socialiste, catholique, libéral, écologistes...) et les communautés linguistiques (francophone et néerlandophone). Aussi, les parlementaires des partis de la majorité tendent à s'aligner sur les compromis établis au sein de la coalition gouvernementale, en dépit des frustrations qui peuvent évidemment naître de la faiblesse de leur marge de manœuvre face aux projets de loi (De Winter, 1996; Deschouwer, 2012). La discussion relative à l'évaluation législative révèle une autre ligne de conflit, celle qui oppose la Chambre des représentants et le Sénat, elle-même reliée à la question des équilibres communautaires entre le nord et le sud du pays.

Le Sénat belge fut conçu, lors de la rédaction de la Constitution de 1831, comme une institution conservatrice, destinée à modérer l'action de la Chambre des représentants et dotée de pouvoirs quasi équivalents. Le mouvement de fédéralisation du pays initié dans les années 1970 a toutefois restreint son influence (Istasse, Sägesser, 2014). Aux termes de la réforme constitutionnelle de 1993, le Sénat devient une « chambre des communautés », composée de représentants des entités fédérées et aux compétences réduites. À la suite de la sixième réforme de l'État (2014), il ne conserve pleinement son rôle de co-législateur que sur les questions institutionnelles. Mais parallèlement, il est redéfini en 1993 comme une «chambre de réflexion », un forum démocratique où les grands enjeux sociaux sont débattus de manière sereine, loin de l'urgence caractérisant les travaux de la Chambre des Représentants. S'ouvre alors une phase de lutte sur le sens exact de la fonction du Sénat, lutte qui continue encore aujourd'hui. Or, ces controverses viennent interférer avec le processus d'institutionnalisation de l'évaluation législative. Députés et sénateurs se disputent en effet la compétence d'évaluation, illustrant par là même sa dimension stratégique.

Ainsi, le député Geert Bourgeois, défendant son idée d'un collège législatif, estime naturel de le rattacher à la Chambre : «Le collège dépendra de la Chambre des représentants, ce qui soulignera la primauté du législateur. Étant donné que le Sénat est appelé à devenir avec le temps un Sénat des communautés, le conseil législatif sera un organe de la Chambre des représentants ${ }^{16}$. L'argument, repris par d'autres députés, est que la Chambre, étant désormais le principal législateur, est la plus à même de réaliser l'évaluation des lois qu'elle vote; le Sénat, représentant les intérêts des institutions fédérées et aux compétences résiduelles, n'aurait pas vocation à critiquer la législation fédérale. Les sénateurs mettent quant à eux l'accent sur l'autre mission qui lui est attribuée, celle de chambre de réflexion, pour justifier la mise en place d'une cellule d'évaluation dans leurs services. À l'occasion de l'ouverture de la session ordinaire 1997-1998, le président du Sénat, spéculant sur l'avenir de l'institution, énonce ainsi qu'il « occupera une place centrale dans le processus d'évaluation législative. Cette option est conforme à l'esprit de la dernière réforme des institutions, qui a fait du Sénat la chambre de réflexion par excellence $»^{17}$. Cette position est alors approuvée par le gouvernement, le ministre de l'Intérieur Louis Tobback (socialiste flamand) déclarant à l'occasion de l'examen de la proposition Vandenberghe : « Le gouvernement tient néanmoins à souligner le rôle spécifique du Sénat. En sa qualité de chambre de réflexion, le Sénat est appelé à examiner les rapports de manière approfondie. Le gouvernement reconnaît ainsi le rôle du Sénat en tant que centre d'évaluation de la législation $»^{18}$.

\footnotetext{
16 Ibid., p. 9.

17 Doc. Sénat, 1-132, 14 octobre 1997, p. 3541.

${ }^{18}$ Doc. Sénat, 1-955/1, 14 janvier 1999, p. 30.
} 
La structure bicamérale du Comité de suivi législatif résulte donc d'une conciliation des revendications des deux assemblées. Mais de ce fait, le destin de l'évaluation législative se trouve déterminé par celui, actuellement fort incertain, du Sénat. Ce dernier, après la sixième réforme de l'État, cherche encore à stabiliser ses nouvelles règles de fonctionnement et à convaincre de son utilité. Son existence même est contestée par un des piliers de la coalition au pouvoir, la N-VA (nationalistes flamands), pour qui l'indépendance de la Flandre passe par le démantèlement de cette « chambre des communautés ». La N-VA pratique en conséquence une politique d'obstruction visant à étouffer toute initiative à même de donner un nouveau souffle à la Haute Assemblée (Istasse, 2015). Le Comité de suivi législatif, organe mineur, risque d'être parmi les victimes de cette stratégie. Il ne s'est pas d'ailleurs pas réuni depuis juillet 2014, date d'installation du Sénat «nouvelle mouture ». Les évolutions récentes semblent donc devoir conduire à un étiolement de l'évaluation parlementaire des lois en Belgique. Un tel résultat n'est pas bien sûr sans rappeler les mésaventures initiales de l'évaluation législative en France. L'Office parlementaire d'évaluation des politiques publiques et l'Office parlementaire d'évaluation de la législation, instances bicamérales créées simultanément en 1996, ont ainsi été paralysés par les rivalités entre les chambres, rivalités encore aggravées du fait du changement de majorité à l'Assemblée après les élections législatives de 1997 (Duprat, 1998; Lacouette-Fougère et Lascoumes, 2013; Perret, 2014). Le premier de ces Offices fut en conséquence supprimé dès 2001 ; le deuxième s'est maintenu officiellement jusqu'en 2009, mais en n'ayant produit en tout que trois rapports. Toutefois, l'évaluation parlementaire française a trouvé un second souffle grâce à l'institution dès 1999 d'une Mission d'Évaluation et de Contrôle relevant uniquement de l'Assemblée nationale. Une opportunité comparable pourrait être offerte à l'évaluation législative belge par une relocalisation à la Chambre des représentants, solution à laquelle les nationalistes flamands pourraient consentir (c'était en effet le sens de la proposition du député N-VA Geert Bourgeois).

\section{Conclusion : quelle effectivité de l'évaluation législative?}

Au terme de cette analyse, les progrès de l'évaluation législative en Belgique apparaissent réels mais inégaux : si les procédures permettant une évaluation ex ante systématique sont en place, l'évaluation ex post est quant à elle entravée par une conception restrictive des critères d'évaluation et par les controverses institutionnelles intercommunautaires. Or, poser la question de l'institutionnalisation d'un dispositif juridique amène inévitablement à débattre de son effectivité : les procédures mises en place sont-elles respectées? Les évaluations produites correspondent-elles aux standards voulus? Les résultats des évaluations sontils appropriés par le personnel politique et ont-ils un impact sur la législation? Quelques constats peuvent être faits à cet égard à partir des rapports des organes d'évaluation en charge de l'évaluation législative.

Concernant les évaluations ex post, il a été indiqué que le Comité de suivi législatif ne s'est réuni que durant trois ans et que durant cette période, seules trois lois correctrices ont été élaborées. Le rapport d'activité 2013/2014 du Comité indique par ailleurs de manière lapidaire que deux des requêtes déposées par des citoyens devaient donner lieu à une «évaluation approfondie ${ }^{19}$, mais celles-ci n'ont pas été réalisées. Le même rapport suggère

\footnotetext{
${ }^{19}$ Doc. Chambre, 53-1963/3, p. 12.
} 
que les séances du Comité ont été essentiellement consacrées au suivi de la jurisprudence de la Cour constitutionnelle, tâche importante mais ne relevant que de la qualité formelle des textes au détriment de leur efficacité. Il semble de plus que très peu de parlementaires se soient associés régulièrement à ces délibérations, ou même en aient été informés. La révolution que devait constituer l'institutionnalisation de l'évaluation législative dans les assemblées ne s'est donc pas manifestée.

Mais les évaluations ex ante pilotées par l'exécutif, en apparence plus structurées et systématisées, ne sont pas non plus exemptes de défauts. Les travers de l'application du test Kafka mentionnés plus haut, en effet, persistent encore aujourd'hui. Les rapports du Comité d'analyse d'impact montrent ainsi que le bilan de l'application de la loi du 15 décembre 2013 est mitigé. Sur le plan quantitatif, tout d'abord : sur les 98 avant-projets de loi approuvés par le Conseil des Ministres en 2014, seuls $73 \%$ étaient pourvus d'une AIR. Les chiffres équivalents pour l'année 2015 sont encore moins souriants : sur 208 avant-projets de loi approuvés, seuls $38 \%$ étaient accompagnés d'une AIR.

Les mêmes rapports mettent également en avant des problèmes d'ordre qualitatif, au niveau du contenu, de la temporalité et de la diffusion des AIR. Un grand nombre d'analyses seraient ainsi insuffisamment approfondies, les développements étant superficiels, stéréotypés ou absents. Le CAI souligne à cet égard la fréquente carence de données budgétaires ou statistiques pourtant aisément disponibles. Par exemple, les fonctionnaires ignoreraient souvent les indicateurs ventilés par genre, bien que la production de ceux-ci ait été systématisée dans le cadre des politiques de Gender mainstreaming (le rapport pour l'année 2014 mentionne le chiffre de $7 \%$ d'AIR employant des indicateurs de genre). Plus fondamentalement, le sens de l'exercice serait mal compris, les administrations confondant la notion d'impact réel des textes avec leurs objectifs officiels, oubliant les effets secondaires et négligeant de proposer des mesures correctrices. Ce défaut de réflexivité rend évidemment le document peu utile à l'exécutif comme au législateur. Un manque d'anticipation est également souligné : les AIR seraient rédigées en fin de processus de rédaction des projets de lois ou d'arrêtés, et communiquées au Parlement à un moment où les arbitrages déjà opérés au sein du gouvernement ne peuvent plus être remis en cause. Une étude réalisée en 2015 par la société IDEA Consult pour le compte de la Fédération des Entreprises de Belgique aboutissait à une conclusion similaire : «Les AIR sont réalisées trop tard dans le processus et sont dès lors à peine prises en compte (comme c'était le cas précédemment avec le test Kafka). Résultat : l'AIR devient en effet un "ajout" formel qui ne peut fournir que peu d'informations pour la véritable politique » (FEB, 2015 : 64). Dans ces circonstances, conclut un des rapports du CAI, la fonction des AIR risque de se limiter à «justifier une décision a posteriori » (CAI, 2015 : 22). Enfin, les rapports du CAI regrettent que les études ne soient pas mieux partagées. Elles sont certes publiées sur le site de l'Agence de simplification administrative, et, lorsqu'il s'agit d'un avant-projet de loi, annexé aux documents parlementaires. Mais la diffusion auprès des partenaires sociaux et auprès des organes étatiques engagés dans le travail législatif (Inspection des Finances, Conseil d'État, Cour des Comptes) est décrite comme sporadique, et l'information apportée par les AIR n'est pas reflétée dans les questions parlementaires ou les amendements du législateur. Ces constats pessimistes quant à l'effectivité des AIR confirment d'ailleurs l'hypothèse formulée plus haut de la dimension « rituelle» ou «symbolique » de ces dernières en Belgique. 
Mais la nature même du système politique belge a également à voir avec ce défaut d'appropriation des résultats des évaluations. La polity belge est notoirement fragmentée et consensuelle (Deschouwer, 2012). La constitution des coalitions y passe par la négociation d'accords de gouvernement dont la conclusion peut prendre plusieurs mois (541 jours après les élections de juin 2010 !). Dans ce contexte, le premier ministre n'a pas l'autorité suffisante pour imposer un modèle d'évaluation systématique et rigoureux, ni d'ailleurs d'incitation à le faire. Les évaluations sont même potentiellement une entrave à la décision, dans un processus entièrement structuré par l'équilibre entre formations politiques. Le cas belge illustre donc plus particulièrement la fragilité de l'ambition de l'evidence-based policy-making (dont l'évaluation est un des piliers) à rationaliser le processus législatif : le maintien du consensus entre partis, garant de la gouvernabilité du pays, y est toujours prioritaire face à la démarche scientifique des évaluateurs ou autres experts.

Cette situation restreint également l'intérêt que peut représenter l'évaluation législative pour le Parlement. Les projets de loi qui y sont déposés sont déjà le résultat de compromis antérieurs entre partis au pouvoir; il est difficile pour les députés et sénateurs de les amender, sauf à la marge, et ce même s'ils s'appuient sur les résultats d'une évaluation. Il n'y a dès lors guère de bénéfice, pour les élus ou les ministres, à s'emparer de la procédure de l'analyse d'impact ou à s'investir personnellement dans un organe tel que le Comité de suivi législatif. Ces obstacles à l'institutionnalisation de l'évaluation législative dans le pays ne semblent pas devoir être levés dans les années à venir, la classe politique belge apparaissant comme de plus en plus divisée et le rôle des assemblées (particulièrement le Sénat) fragilisé. 


\section{Bibliographie}

Bergel J.-L., 1995. Problématique et méthode de l'évaluation législative In Jean-Louis Bergel (dir.), Contrôle parlementaire et évaluation, Paris, La Documentation française, 193-200.

Bouckaert G. and Pollitt C., 2011. Public Management Reform. A Comparative Analysis. Oxford Press University, Oxford.

Bussmann W., 2010. Evaluation of Legislation: Skating on thin Ice. Evaluation 16 (3), 279-293.

Cerexhe G., 2001. L'évaluation des lois In Christian de Visscher, Frédéric Varone (dir.), Évaluer les politiques publiques. Regards croisés sur la Belgique, Academia-Bruylant, Louvain-La-Neuve, 117-132.

Chevallier J., 1995. L'évaluation législative: un enjeu politique In Jean-Louis Bergel (dir.), Contrôle parlementaire et évaluation, La Documentation française, Paris, 13-27.

Commission Européenne, 2001. Communication: Simplifying and Improving the Regulatory Environment. Brussels.

Debeuckelaere K. et Kegels C., 2000. L'analyse d'impact de la réglementation (AIR) : un outil pour améliorer la prise de décision politique? Pyramide 2, 111-120.

De Peuter B., Pattyn V. and Speer S., 2015. The Growing Role of Evaluation in Parliaments: Holding Governments Accountable? International of Administrative Science 81 (1), 37-57.

Deschouwer K., 2012. The Politics of Belgium. Governing a Divided Society. Palgrave McMillan, Basingstoke.

De Winter L., 1996. Party encroachment on executive and legislative branch in the Belgian polity. Res Publica 38 (2), 325-352.

Dolowitz D. and Marsh D., 2000. Learning from Abroad. The Role of Policy Transfer in Contemporary PolicyMaking. Governance 1, 5-24.

Duprat J.-P., 1998. Le parlement évaluateur. Revue internationale de droit comparé 50 (2), 551-576.

FEB, 2015. La qualité de la réglementation en Belgique : Un obstacle ou un levier pour la compétitivité? Bruxelles.

Flückiger A., 2008. L'évaluation législative ou comment mesurer l'efficacité des lois. Revue européenne des sciences sociales 141, 83-101.

Furubo J.-E., Rist R.-E. and Sandahl R., 2002. International Atlas of Evaluation. Transaction Publishers, New Brunswick.

Furubo J.-E., Jacob S. and Speer S., 2015. The institutionalization of evaluation matters: Updating the International Atlas of Evaluation 10 years later. Evaluation 21 (1), 6-31.

Istasse C. and Sägesser C., 2014. Le Sénat et ses réformes successives. Courrier hebdomadaire du CRISP 22192220.

Istasse C., 2015. Quel devenir pour le Sénat? Premier bilan. Les analyses du CRISP en ligne, 21 mai 2015, www. crisp.be

Jacob S., 2005. La volonté des acteurs et le poids des structures dans l'institutionnalisation de l'évaluation des politiques publiques (France, Belgique, Suisse et Pays-Bas). Revue française de science politique 55 (5), 835-864.

Karpen U., 2002. Evaluation of legislation. Nomos, Baden-Baden.

Karpen U., 2004. Implementation of legislative evaluation in Europe. Current models and trends. European Journal of Law Reform 6 (1-2), 57-85.

Lacouette-Fougere C. et Lascoumes P., 2013. L'évaluation : un marronnier de l'action gouvernementale? Revue française d'administration publique 148, 859-875.

Lacroix B. et Lagroye J. (dir.), 1992. Le Président de la république. Génèse et usages d'une institution. Presses de la Fondation Nationale des Sciences Politiques, Paris.

Mabille X., 1990. Le débat politique d'avril 1990 sur la sanction et la promulgation de la loi sur l'IVG. Courrier hebdomadaire du CRISP 1275. 
Mader L., 2001. Evaluating the Effects: A Contribution to the Quality of Legislation. Statute Law Review 22 (2), 119-31.

Meny Y. et Thoenig J.-C., 1987. Politiques publiques. PUF, Paris.

OCDE, 1997. L'analyse de l'impact de la réglementation: Meilleures pratiques dans les pays de l'OCDE. OCDE, Paris.

OCDE, 2010. Better regulation in Europe : Belgium. OCDE, Paris.

Perret C., 2014. L'évaluation des politiques publiques. La Découverte, Paris.

Radaelli C., 2009. Rationality, Power, Management and Symbols: Four images of Regulatory Impact Assessment. Scandinavian Political Studies 2, 164-188.

Radaelli C., 2010. Regulating rule-making via impact assessment. Governance 1, 89-108.

Radaelli C. and De Francesco F., 2010. Regulatory Impact Assessment In Baldwin R, Oxford Handbook of Regulation, Oxford University Press, Oxford, 279-301.

Swennen B., 2001. L'évaluation de la politique d'interruption volontaire de grossesse In Christian de Visscher, Frédéric Varone (dir.), Évaluer les politiques publiques. Regards croisés sur la Belgique, Académia-Bruylant, Louvain-la-Neuve, 103-114.

Varone F., Jacob S. et De Winter L., 2005. Polity, politics and policy evaluation in Belgium. Evaluation 11 (3), 253-273.

Zwaan et al., 2016. Évaluation législative ex post dans l'Union européenne : questionnement de l'utilisation d'évaluations en tant qu'instruments d'imputabilité. Revue Internationale des Sciences Administratives 4, 713-732. 